Draft VERSion FEBruary 6, 2020

Typeset using IATEX RNAAS style in AASTeX63

\title{
Potential Backup Targets for Comet Interceptor
}

\author{
Megan E. Schwamb (D), ${ }^{1}$ Matthew M. Knight (D), ${ }^{2,3}$ Geraint H Jones (D), ${ }^{4,5}$ Colin Snodgrass (D), ${ }^{6}$ Lorenzo Bucci, ${ }^{7}$ \\ José Manuel Sánchez Pérez, ${ }^{7}$ Nikolai Skuppin ${ }^{7}$ \\ for the Comet Interceptor Science Team

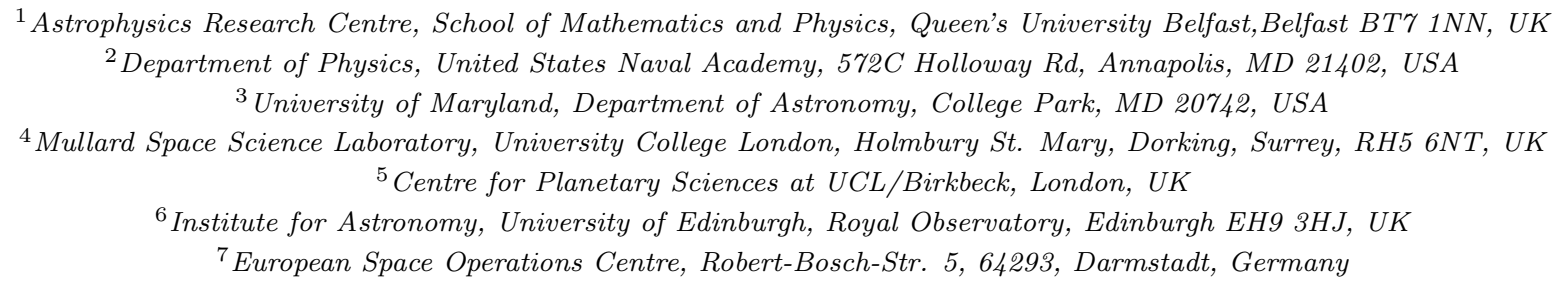

\section{COMET INTERCEPTOR MISSION}

Comet Interceptor (Snodgrass \& Jones 2019) is an ESA (European Space Agency) F-class (Fast) mission expected to launch in 2028 on the same launcher as ESA's ARIEL (Atmospheric Remote-sensing Infrared Exoplanet Large-survey; Tinetti et al. 2016) mission. Comet Interceptor's science payload consists of three spacecraft, a primary spacecraft that will carry two smaller probes to be released at the target. The three spacecraft will fly-by the target along different chords, providing multiple simultaneous perspectives of the comet nucleus and its environment. Each of the spacecraft will be equipped with different but complementary instrument suites designed to study the far and near coma environment and surface of a comet or interstellar object (ISO), a rogue planetesimal formed around another star system passing through the Solar System The primary spacecraft will perform a fly-by at $\sim 1000 \mathrm{~km}$ from the target. The two smaller probes will travel deeper into the coma, closer to the nucleus.

The mission is being designed and launched without a specific comet designated as its main target. Comet Interceptor will travel to the Sun-Earth L2 Lagrangian point with ARIEL and wait in hibernation until a suitable long-period comet (LPC) is found that will come close enough to the Sun for the spacecraft to maneuver to an encounter trajectory. Ideally, the mission will visit a dynamically new comet (DNC), a LPC on its first entry into the the inner Solar System from the Oort cloud, or an ISO. Since 1970, $23 \%$ of LPCs discovered with $\mathrm{q}<3.1$ au are DNCs (Królikowska \& Dybczyński 2019). Comet Interceptor's encounter target will mostly likely be discovered in the 2020s with the onset of the Vera C. Rubin Observatory's Legacy Survey of Space and Time (LSST; Ivezić et al. 2019). Over its 10-year baseline survey, LSST is expected to find $\sim 10,000$ new comets (Solontoi et al. 2010) and to discover several ISOs per year (e.g., Cook et al. 2016; Rice \& Laughlin 2019).

\section{REQUEST FOR COMMUNITY OBSERVATIONS}

In the event of a suitable LPC or ISO not being discovered before Comet Interceptor is required to leave L2, the science team will select a known short-period comet as the mission target. To prepare for all eventualities, the science team has assembled a preliminary set of backup targets from the known Jupiter family comets (see Table 1), where a suitable fly-by trajectory can be achieved during the nominal mission timeline (including the possibility of some launch delay). To better prioritize this list, we are releasing our potential backup targets in order to solicit the planetary community's help with observations of these objects over future apparitions and to encourage publication of archival data on these objects. Observations characterizing the nuclei of these comets (size, shape, rotation period) and their activity levels (especially around perihelion) would be particularly useful. Astrometry and improved orbits, especially for the more recently discovered or fragmented comets would also be beneficial. Any additional information that may assist scientific prioritization of these targets would be welcomed by the Comet Interceptor team.

Corresponding author: Megan E. Schwamb

m.schwamb@qub.ac.uk 
Table 1. Potential Comet Interceptor Backup Targets

\begin{tabular}{|c|c|c|c|c|c|c|}
\hline $\begin{array}{c}\text { MPC } \\
\text { Designation }\end{array}$ & $\begin{array}{c}\text { Orbital } \\
\text { Period } \\
\text { (yrs) }\end{array}$ & $\begin{array}{c}\mathrm{q} \\
(\mathrm{au})\end{array}$ & $\begin{array}{c}\mathrm{e} \\
(\mathrm{deg})\end{array}$ & $\begin{array}{c}\mathrm{i} \\
(\mathrm{deg})\end{array}$ & $\begin{array}{c}\text { Fly-by } \\
\text { date } \\
\text { DD-MM-YYYY }\end{array}$ & Notes \\
\hline P/2016 BA14 (PANSTARRS) & 5.25 & 1.01 & 0.666 & 18.92 & $20 / 04 / 2037$ & May have recently split $(\text { from } 252 \mathrm{P})^{A}$ \\
\hline 7P/Pons-Winnecke & 6.37 & 1.26 & 0.634 & 22.29 & $28 / 09 / 2033$ & \\
\hline $8 \mathrm{P} /$ Tuttle & 13.61 & 1.03 & 0.820 & 54.98 & $26 / 03 / 2035$ & Contact binary; Oort cloud source? ${ }^{B}$ \\
\hline 15P/Finlay & 6.51 & 0.97 & 0.720 & 6.80 & $18 / 09 / 2034$ & \\
\hline 21P/Giacobini-Zinner & 6.54 & 1.01 & 0.710 & 32.00 & $07 / 09 / 2031$ & $\begin{array}{l}\text { Encountered by the International } \\
\text { Cometary Explorer }(I C E ; 1985)^{C}\end{array}$ \\
\hline 26P/Grigg-Skjellerup & 5.31 & 1.12 & 0.633 & 22.36 & $10 / 06 / 2034$ & Encountered by Giotto $(1992)^{D}$ \\
\hline 73P/Schwassmann-Wachmann 3 & 5.44 & 0.97 & 0.686 & 11.24 & $05 / 04 / 2033$ & Multiple nucleus fragments \\
\hline 189P/NEAT & 4.98 & 1.17 & 0.598 & 20.40 & $01 / 09 / 2032$ & 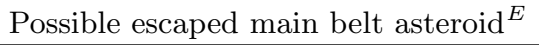 \\
\hline 289P/Blanpain & 5.32 & 0.96 & 0.685 & 5.90 & $23 / 11 / 2035$ & Tiny, almost extinct ${ }^{F}$ \\
\hline 300P/Catalina & 4.42 & 0.82 & 0.694 & 5.70 & $19 / 06 / 2036$ & Possible escaped main belt asteroid $^{E}$ \\
\hline \multicolumn{7}{|l|}{$A_{\text {Li et al. }}(2017)$} \\
\hline \multicolumn{7}{|l|}{$B_{\text {Harmon et al. (2010) }}$} \\
\hline \multicolumn{7}{|l|}{$C_{\text {Ogilvie et al. (1986) }}$} \\
\hline \multicolumn{7}{|l|}{$D_{\text {Grensemann \& Schwehm (1993) }}$} \\
\hline \multicolumn{7}{|l|}{$E_{\text {Fernández \& Sosa }}(2015)$} \\
\hline$F_{\text {Jewitt }}(2006)$ & & & & & & \\
\hline
\end{tabular}

Heliocentric orbital elements taken from JPL Small-Body Database Search Engine https://ssd.jpl.nasa.gov/sbdb_query.cgi\# queried on 28 January 2020.

\section{REFERENCES}

Cook, N. V., Ragozzine, D., Granvik, M., \& Stephens, D. C. 2016, ApJ, 825, 51, doi: 10.3847/0004-637X/825/1/51

Fernández, J. A., \& Sosa, A. 2015, Planet. Space Sci., 118, 14, doi: 10.1016/j.pss.2015.07.010

Grensemann, M. G., \& Schwehm, G. 1993,

J. Geophys. Res., 98, 20907, doi: 10.1029/93JA02528

Harmon, J. K., Nolan, M. C., Giorgini, J. D., \& Howell, E. S. 2010, Icarus, 207, 499, doi: $10.1016 /$ j.icarus.2009.12.026

Ivezić, Ž., Kahn, S. M., Tyson, J. A., et al. 2019, ApJ, 873, 111, doi: 10.3847/1538-4357/ab042c

Jewitt, D. 2006, AJ, 131, 2327, doi: 10.1086/500390

Królikowska, M., \& Dybczyński, P. A. 2019, MNRAS, 484, 3463, doi: 10.1093/mnras/stz025
Li, J.-Y., Kelley, M. S. P., Samarasinha, N. H., et al. 2017, AJ, 154, 136, doi: 10.3847/1538-3881/aa86ae

Ogilvie, K. W., Coplan, M. A., Bochsler, P., \& Geiss, J. 1986, Science, 232, 374, doi: 10.1126/science.232.4748.374

Rice, M., \& Laughlin, G. 2019, ApJL, 884, L22, doi: $10.3847 / 2041-8213 / a b 4422$

Snodgrass, C., \& Jones, G. H. 2019, Nature Communications, 10, 5418, doi: 10.1038/s41467-019-13470-1

Solontoi, M., Ivezić, Ž., West, A. A., et al. 2010, Icarus, 205, 605, doi: 10.1016/j.icarus.2009.07.042

Tinetti, G., Drossart, P., Eccleston, P., et al. 2016, Society of Photo-Optical Instrumentation Engineers (SPIE) Conference Series, Vol. 9904, The science of ARIEL (Atmospheric Remote-sensing Infrared Exoplanet Large-survey), 99041X, doi: 10.1117/12.2232370 


\section{ACKNOWLEDGMENTS}

This work has made use of NASA's Astrophysics Data System Bibliographic Services. MES was supported by STFC Grant ST/P000304/1. 